\title{
Correlations in emitters coupled to plasmonic waveguides
}

\author{
C E Susa ${ }^{1 \dagger}$, J H Reina ${ }^{1}$ and L L Sánchez-Soto ${ }^{2}$ \\ ${ }^{1}$ Departamento de Física, Universidad del Valle, A. A. 25360, Cali, Colombia \\ 2 Departamento de Óptica, Facultad de Física, Universidad Complutense, 28040 Madrid, \\ Spain \\ E-mail: ${ }^{\dagger}$ cristian.susa.q@correounivalle.ed.co
}

\begin{abstract}
We report on quantum, classical, and total correlations in a set of distant quantum emitters coupled via their interaction with the plasmon modes of a one-dimensional waveguide driven by an external laser field. The coupling of the emitters with the plasmonic modes and its influence on the collective decay rate suggest that entanglement does not play a significant role in the qubit dynamics. Rather, discord is the quantity that matters and should be harnessed as a resource.
\end{abstract}

PACS numbers: 03.65.Ud, 03.67.Lx, 42.79.Gn

\section{Introduction}

Entanglement is a cornerstone of quantum theory [1-4]. It has strong practical implications for futuristic quantum technologies [5-10] and, consequently, it has been investigated in a wide variety of physical systems [11-14]. For a thorough description of this phenomenon, a formal framework has been introduced over the past decade to quantify quantum [15] and classical [16] correlations in a physical system. The former, being of a more general character than entanglement, has also been hinted to play a role in quantum information processing through the so-called mixed state-based quantum computing [17, 18].

Quantum emitters (e.g., single molecules [19,20] or quantum dots [21-23]) coupled to surface plasmons of conducting nanowires or waveguides have been seen as a promising hardware for quantum information processing because of the strong coupling that can be achieved between the emitters and the plasmons [21]. The collective spontaneous decay and the plasmon-mediated emitter-emitter coupling have been investigated for a two-qubit (emitter) system close to a nanowire [24, 25], as has the resonance energy transfer mediated by different plasmonic nanowaveguides [26]. Entanglement of two qubits, mediated by a V-groove plasmonic waveguide, has recently been theoretically studied [27, 28] and quantified via the concurrence [29]. We address this problem within a more general framework: we calculate the entire spectrum for the entanglement, classical, quantum, and total correlations [30-32] for emitters coupled to plasmonic modes.

We demonstrate that it is not entanglement but the quantum correlations (measured by the discord) that provide the relevant robust features that could be harnessed for plasmonassisted information processing. Our results support the conclusion that the entanglement of formation [29] is a natural metric and that, in the context of entropic measures, quantification via the concurrence might be inappropriate. 


\section{Quantum emitters, plasmon modes and correlations}

We start by briefly delineating the model. We are dealing with two two-level atoms fixed at positions $\mathbf{r}_{i}(i=1,2)$, with transition frequencies $\omega_{i}$ and separated by the vector $\mathbf{r}_{12}$. We denote by $\left|0_{i}\right\rangle$ and $\left|1_{i}\right\rangle$ the ground and excited states of the emitter $i$, with associated transition dipole moments $\hat{\mu}_{i} \equiv\left\langle 0_{i}\left|\mathbf{D}_{i}\right| 1_{i}\right\rangle, \mathbf{D}_{i}$ being the corresponding dipole operators.

The emitters are embedded in a medium of refractive index $n$ and interact via a dipoledipole coupling, so that the system Hamiltonian $H_{S}$ can be written as

$$
H_{S}=H_{0}+H_{12},
$$

where the free and interaction Hamiltonians are

$$
\begin{aligned}
& H_{0}=\frac{1}{2} \hbar \omega_{1} \sigma_{z}^{(1)}+\frac{1}{2} \hbar \omega_{2} \sigma_{z}^{(1)} \\
& H_{12}=\frac{1}{2} \hbar V\left(\sigma_{x}^{(1)} \otimes \sigma_{x}^{(2)}+\sigma_{y}^{(1)} \otimes \sigma_{y}^{(2)}\right) .
\end{aligned}
$$

The strength $V$ depends of the configuration of the interacting dipoles and $\sigma^{(i)}$ are the Pauli operators.

In addition, an external laser field of frequency $\omega_{L}$ drives the emitters, which we represent by

$$
H_{L}=\hbar \ell^{(1)}\left(\sigma_{-}^{(1)} e^{i \omega_{L} t}+\sigma_{+}^{(1)} e^{-i \omega_{L} t}\right)+\hbar \ell^{(2)}\left(\sigma_{-}^{(2)} e^{i \omega_{L} t}+\sigma_{+}^{(2)} e^{-i \omega_{L} t}\right) .
$$

Here $\ell^{(i)}$ is the strength of this coupling, and is given by $\hbar \ell^{(i)}=-\hat{\mu}_{i} \cdot \mathbf{E}_{i}$, with $\mathbf{E}_{i}$ being the amplitude of the coherent field acting on the $i$ th emitter, and $\sigma_{+}^{(i)}=\left|1_{i}\right\rangle\left\langle 0_{i}\right|$, and $\sigma_{-}^{(i)}=\left|0_{i}\right\rangle\left\langle 1_{i}\right|$ are the raising and lowering Pauli operators acting on the $i$ th emitter.

\subsection{Collective decay effects}

We next assume that the emitters are coupled with a bath of the radiation field, so that the corresponding dissipative dynamics is given by the total Hamiltonian $H=H_{S}+H_{L}$, by means of the quantum master equation [20]

$$
\begin{aligned}
\dot{\rho} & =-\frac{i}{\hbar}[H, \rho] \\
& -\sum_{i, j=1}^{2} \frac{\Gamma_{i j}}{2}\left(\rho \sigma_{+}^{(i)} \sigma_{-}^{(j)}+\sigma_{+}^{(i)} \sigma_{-}^{(j)} \rho-2 \sigma_{-}^{(i)} \rho \sigma_{+}^{(j)}\right),
\end{aligned}
$$

Without loss of generality, we set $\Gamma_{i i} \equiv \Gamma_{i}=\Gamma$, and $\Gamma_{i j}=\Gamma_{j i}^{*} \equiv \gamma(i \neq j)$, the individual, and collective spontaneous emission rates, respectively.

The explicit form of the individual emitter decay rate $\Gamma_{i}$, as well as the collective decay rate $\gamma$ associated to the dipole-dipole (qubit-qubit) interaction $V$ depend on the particular physical setup under consideration. For the case of interacting 'bare' quantum emitters, we have

$$
\begin{aligned}
V & =\frac{3 \sqrt{\Gamma_{1} \Gamma_{2}}}{4}\left\{\hat{\mu}_{1} \cdot \hat{\mu}_{2}-\left(\hat{\mu}_{1} \cdot \hat{\mathbf{r}}_{12}\right)\left(\hat{\mu}_{2} \cdot \hat{\mathbf{r}}_{12}\right)\right] \frac{\cos z}{z} \\
& \left.+\left[\hat{\mu}_{1} \cdot \hat{\mu}_{2}-3\left(\hat{\mu}_{1} \cdot \hat{\mathbf{r}}_{12}\right)\left(\hat{\mu}_{2} \cdot \hat{\mathbf{r}}_{12}\right)\right]\left(\frac{\cos z}{z^{3}}+\frac{\sin z}{z^{2}}\right)\right\},
\end{aligned}
$$




$$
\begin{aligned}
\gamma & =\frac{3 \sqrt{\Gamma_{1} \Gamma_{2}}}{2}\left\{\left[\hat{\mu}_{1} \cdot \hat{\mu}_{2}-\left(\hat{\mu}_{1} \cdot \hat{\mathbf{r}}_{12}\right)\left(\hat{\mu}_{2} \cdot \hat{\mathbf{r}}_{12}\right)\right] \frac{\sin z}{z}\right. \\
& \left.+\left[\hat{\mu}_{1} \cdot \hat{\mu}_{2}-3\left(\hat{\mu}_{1} \cdot \hat{\mathbf{r}}_{12}\right)\left(\hat{\mu}_{2} \cdot \hat{\mathbf{r}}_{12}\right)\right]\left(\frac{\cos z}{z^{2}}-\frac{\sin z}{z^{3}}\right)\right\},
\end{aligned}
$$

where $z=n k_{0} r_{12}, k_{0}=\omega_{0} / c$, and $\omega_{0}=\left(\omega_{1}+\omega_{2}\right) / 2$.

We next consider that the emitters are located close to a plasmonic waveguide. The new emission properties, as well as their dipolar interaction strength, depend on the coupling to the plasmonic modes. The dipole-dipole interaction strength and the incoherent decay rate can be calculated from the Green's function $\mathrm{G}\left(\mathrm{r}_{1}, \mathrm{r}_{2}\right)$ that describes the electromagnetic interaction between the two dipole moments (emitters) $\mu_{1,2}$ of frequency $\omega_{1}=\omega_{2} \equiv \omega_{0}$ as follows (see [25] for a discussion of the evaluation of these terms)

$$
\begin{aligned}
V^{\mathrm{pl}} & =\frac{1}{\pi \varepsilon_{0} c^{2} \hbar} \mathscr{P} \int_{0}^{\infty} \frac{\omega^{2} \operatorname{Im}\left[\mu_{1}^{*} \mathrm{G}\left(\omega, \mathbf{r}_{1}, \mathbf{r}_{2}\right) \mu_{2}\right]}{\omega-\omega_{0}} d \omega, \\
\Gamma_{i j}^{\mathrm{pl}} & =\frac{2 \omega_{0}^{2}}{\varepsilon_{0} c^{2} \hbar} \operatorname{Im}\left[\mu_{i}^{*} \mathrm{G}\left(\omega_{0}, \mathbf{r}_{i}, \mathbf{r}_{j}\right) \mu_{j}\right],
\end{aligned}
$$

where the superscript "pl" indicates that the collective parameters are now modified by the interaction with the plasmonic waveguide. As before, we set $\Gamma_{i i}^{\mathrm{pl}}=\Gamma_{i}^{\mathrm{pl}}$, and $\Gamma_{i j}^{\mathrm{pl}}=\left(\Gamma_{j i}^{\mathrm{pl}}\right)^{*}=\gamma^{\mathrm{pl}}$, with $i, j=1,2$.

It has been recently shown [27, 28] that both the dipolar interaction and the collective damping for a pair of emitters close enough to a plasmonic waveguide hold simple analytical forms when the dominant contribution for emission is due to the plasmons. Thus, the Green function can be approximated by the plasmonic Green function contribution, $\mathrm{G}\left(\mathrm{r}_{1}, \mathrm{r}_{2}, \omega\right) \approx$ $\mathrm{G}^{\mathrm{pl}}\left(\mathrm{r}_{1}, \mathrm{r}_{2}, \omega\right)$.

If we set the plasmon wavelength $\lambda_{\mathrm{pl}}=2 \pi / k_{\mathrm{pl}}$, we have [25.27]

$$
V^{\mathrm{pl}}=\frac{1}{2} \Gamma^{\mathrm{pl}} \tilde{\beta} \sin (2 \pi \zeta), \quad \gamma^{\mathrm{pl}}=\Gamma^{\mathrm{pl}} \tilde{\beta} \cos (2 \pi \zeta),
$$

where $\tilde{\beta}=\beta \exp \left[-\lambda_{\mathrm{pl}} \zeta /(2 L)\right]$, with $\zeta=d / \lambda_{\mathrm{pl}}, d$ is the distance between the emitters, $\Gamma^{\mathrm{pl}}=\Gamma_{1}^{\mathrm{pl}}=\Gamma_{2}^{\mathrm{pl}}, L$ is the propagation length of the propagating mode, and the $\beta$ factor that measures the fraction of emitted radiation by the propagating mode. It is worth mentioning that, due to radiative contributions, such a 'plasmonic approximation' breaks down for emitters separations shorter than $\sim \lambda_{\mathrm{pl}} / 4$ [25].

We note from these two relations that $\left|V^{\mathrm{pl}}\right| \leq \Gamma^{\mathrm{pl}} / 2$ and $\left|\gamma^{\mathrm{pl}}\right| \leq \Gamma^{\mathrm{pl}}$, which means that the interaction that arises due to the coupling to the plasmons is weak and the most important contribution due to collective effects comes from the damping $\gamma^{\mathrm{pl}}$.

\subsection{Correlations}

The definitions involved in the calculation of the correlations are as follows. The quantum mutual information describes the whole content of correlations in a given quantum system [30-32]. It has been shown that quantum correlations (entanglement included) [15] and classical correlations [16], in the sense of entropic measures, add up to give the quantum mutual information [31]. Furthermore, this point has been recently emphasized, via the use of the relative entropy, within a unified framework that captures both quantum and classical correlations within the quantum mutual information [32]. For a bipartite system, this can be written as:

$$
I\left(\rho_{A B}\right)=S\left(\rho_{A}\right)+S\left(\rho_{B}\right)-S\left(\rho_{A B}\right),
$$

where $S(\rho)=-\operatorname{Tr}\left(\rho \log _{2} \rho\right)$ is the von Neumann entropy of density matrix $\rho$. 
A measure of classical correlations was introduced in Ref. [16] as the maximum extractable classical information from a subsystem, say $A$, when a set of positive operator valued measures [7] has been performed on the other subsystem (say $B$ ):

$$
\mathrm{CC}\left(\rho_{A B}\right)=\sup _{\left\{\Pi_{j}^{B}\right\}}\left[S\left(\rho_{A}\right)-\sum_{j} p_{j} S\left(\rho_{A}^{j}\right)\right],
$$

where $S\left(\rho_{A}^{j}\right)$ is the entropy associated to the density matrix of subsystem $A$ after the measure. Such correlations must be non-increasing, and invariant under local unitary operations, and $\mathrm{CC}\left(\rho_{A B}\right)=0$ if and only if $\rho_{A B}=\rho_{A} \otimes \rho_{B}$.

If $\{|0\rangle,|1\rangle\}$ define the basis states for the qubit $B$, the projectors can be written as $\Pi_{j}^{B}=\mathbf{1} \otimes|j\rangle\langle j|, j=a, b$, where $|a\rangle=\cos \theta|0\rangle+e^{i \phi} \sin \theta|1\rangle,|b\rangle=e^{-i \phi} \sin \theta|0\rangle-\cos \theta|1\rangle$, and the optimization is carried out over angles $\theta$ and $\phi$. The measure CC is antisymmetric by definition, and, without loss of generality, we take the qubit $B$ to be the one measured.

Following the definition for $\mathrm{CC}\left(\rho_{A B}\right)$, a simple way to define the total quantum correlations in a bipartite system is $D\left(\rho_{A B}\right)=I\left(\rho_{A B}\right)-\mathrm{CC}\left(\rho_{A B}\right)$. In terms of the von Neumann entropies, the quantum correlations, which coincide with the definition for the quantum discord given in Ref. [15], read

$$
D\left(\rho_{A B}\right)=S\left(\rho_{B}\right)-S\left(\rho_{A B}\right)+\inf _{\left\{\Pi_{j}^{B}\right\}} \sum_{j} p_{j} S\left(\rho_{A \mid \Pi_{j}^{B}}\right) .
$$

For pure states, $D=S\left(\rho_{B}\right)$, and $D=0$ iff the system is purely classically correlated.

Quantum entanglement is quantified by the entanglement of formation

$$
\operatorname{EoF}(\rho)=h\left(\frac{1+\sqrt{1-C^{2}(\rho)}}{2}\right),
$$

where $h(x)=-x \log _{2} x-(1-x) \log _{2}(1-x)$ denotes the binary entropy function [29].

Additionally, consider, in decreasing order, the eigenvalues $\lambda_{i}$ of the matrix $\sqrt{\rho_{A B} \tilde{\rho}_{A B}}$, where $\tilde{\rho}_{A B}=\left(\sigma_{y} \otimes \sigma_{y}\right) \bar{\rho}_{A B}\left(\sigma_{y} \otimes \sigma_{y}\right)$ and $\bar{\rho}_{A B}$ is the elementwise complex conjugate of $\rho$. The concurrence $C$ can be defined as

$$
C\left(\rho_{A B}\right)=\max \left\{0, \lambda_{1}-\lambda_{2}-\lambda_{3}-\lambda_{4}\right\},
$$

where the $\lambda_{i}$ 's are as introduced above or, equivalently (also in decreasing order), the square root of the eigenvalues of the non-Hermitian matrix $\rho_{A B} \tilde{\rho}_{A B}$ [29].

\section{Dynamics of correlations}

We calculate the exact dynamics of correlations by solving the master equation 2.4, and taking into account the definitions introduced in section 2.2 . The notation for the correlations reported in the graphs throughout this paper is as follows: total correlation or mutual information (purple, thick-solid line), classical correlation (green, doubly-dashed line), quantum discord (blue, thin-solid line), entanglement of formation (red, dashed line), and concurrence (pink, dotted line).

To compare the physical scenarios described in Sec. 2, we first present correlations when the emitters interact solely with the vacuum electromagnetic field, i.e., in the absence of coupling to plasmons. In terms of the collective effects, such a dynamics depends on the separation between emitters and also on the orientation of their dipole moments.

A typical situation where the dipole-dipole interaction is much greater than the incoherent part $V \gg \Gamma \gg \gamma$ [figure 1]a)] can be observed, for example, in a system of diluted 


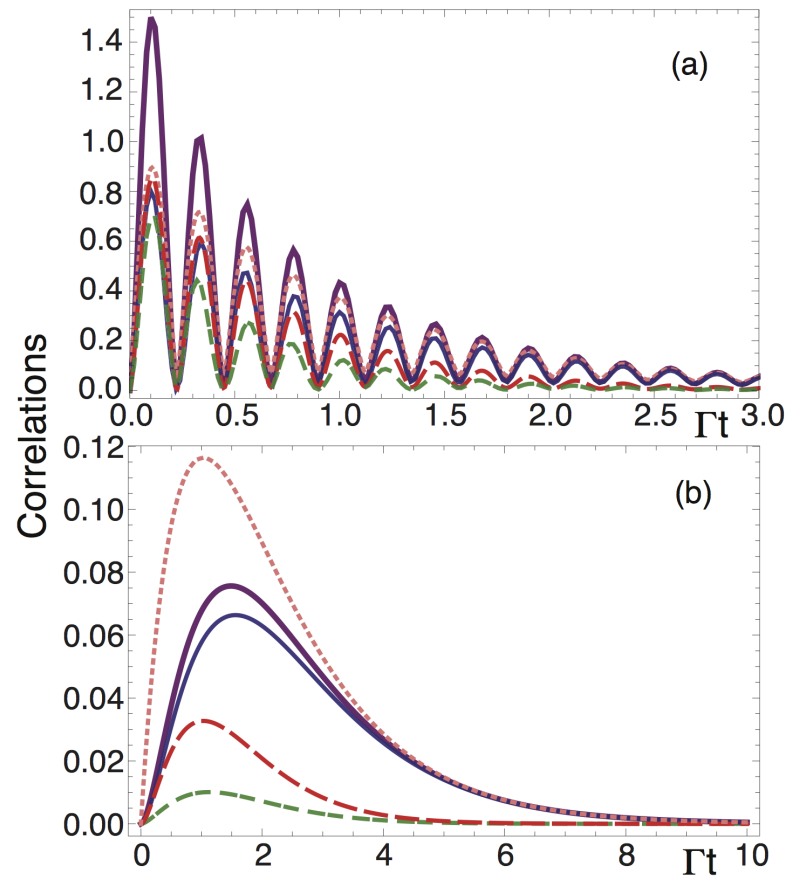

Figure 1. (Color online) Correlations for a two-emitter system in the absence of coupling to plasmons. (a) $V=7 \Gamma$ and $\gamma=0.2 \Gamma$. (b) Dipoles parallel to each other and perpendicular to their separation vector; $r_{12}=\frac{3}{4} \lambda_{0}, \lambda_{0} \equiv 2 \pi / k_{0}$. The considered initial state is separable: $\rho(0)=|10\rangle\langle 10|$. In all graphs presented in this work: Total correlation or mutual information (purple, thick-solid line), classical correlation (green, double-dashed line), quantum discord (blue, thin-solid line), entanglement of formation (red, dashed line), and concurrence (pink, dotted line).

molecules of Terrilene in a dispersive crystal [19], for which a rich entanglement dynamics has been predicted [20]. Figure 1 a) shows that correlations reach high values and oscillate rapidly because of the strong dipole-dipole interaction energy $V$; however, the correlations decay within a short time and the system becomes uncorrelated quite rapidly.

A different scenario, depicted in figure 11 b), considers a pair of dipoles parallel to each other and perpendicular to their separation vector, which allows the calculation of the collective parameters $V$ and $\gamma$ directly from Eqs. 2.5. Although the correlations initially show smaller values than before, they persist for a longer time.

An interesting issue that arises from figure 1 is the discrepancy between the entanglement of formation and the concurrence as quantifiers of entanglement. Figure 1 (b) shows that concurrence reaches much higher values (and decays much slower) than the EoF; more surprising is the fact that concurrence reaches, for almost any time, higher values than the total correlations, a result that contrasts with the definition of the mutual information as a quantity that accounts for all the correlations (classical and quantum), entanglement included [31,32]. Thus, within this framework, concurrence can indicate results that are well above the EoF, and hence does not allow a direct comparison with other entropic measures such as the quantum discord; in contrast, the latter can be compared, on the same grounds, to the EoF [33]. This is explicitly shown in figure 1 (b).

The influence of the plasmonic waveguide is illustrated in figure 2, and can be directly 


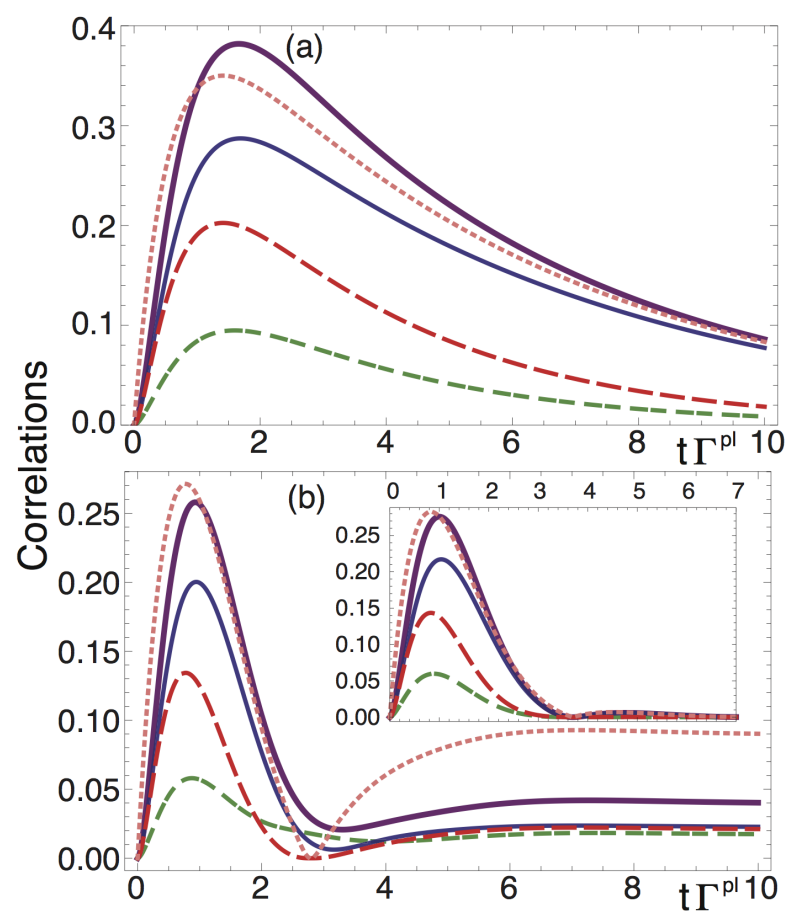

Figure 2. (Color online) Correlations for the emitters interacting with a plasmonic waveguide with the experimentally feasible set of parameters $\beta=0.94, L=2 \mu \mathrm{m}$, obtained for an operational wavelength $\lambda_{0}=640 \mathrm{~nm}$; this gives $\tilde{\beta} \simeq 0.82$, and a plasmonic wavelength $\lambda_{\mathrm{pl}} \simeq 542 \mathrm{~nm}$. (a) Separation between emitters $d=\lambda_{\mathrm{pl}}, V^{\mathrm{pl}}=0$, (b) $\zeta \equiv d / \lambda_{\mathrm{pl}}=3 / 4, \gamma^{\mathrm{pl}}=0$, and $\ell=0.2 \Gamma^{\mathrm{pl}}$. The inset corresponds to case (b), but in the absence of laser pumping. The notations for the graphs are as given in figure 1

compared with the results in figure 1. According to the relations derived from Eqs. 22.6 [25, 27], one of the parameters $V^{\mathrm{pl}}$ or $\gamma^{\mathrm{pl}}$ can, in principle, be maximized by 'switching off' the other one. For the interqubit distance $d=\frac{3}{4} \lambda_{\text {pl }}$ [Fig 2 (b)], the plasmonic modes allow for an effective enhancement of correlations, as can be seen from a direct comparison between the inset of figure 2(b) and figure 1(b). This said, note that the correlations decay more rapidly in the plasmon-assisted case because here the emitters distance is such that the collective rate is switched off, and the nonlocal effects are purely due to the weak dipole-dipole interaction $V^{\mathrm{pl}}$. The correlations are enhanced as $\tilde{\beta}$ tends to 1 . For an emitters separation $d=\lambda_{\mathrm{pl}}$, the realistic value $\tilde{\beta}=0.82$ can be obtained for $L=2 \mu \mathrm{m}$, and this is shown in Fig. 22(a): for this interqubit distance, $V^{\mathrm{pl}}$ is switched off and the correlations reach larger values than before and are maintained for a much longer time. We also point out that for the parameter window considered in figure 2, classical correlations do not play a major role.

Interestingly, the quantum discord, which is in its essence different to entanglement, does play a role (and is more robust than the EoF) during the qubit dissipative evolution. We remark that is not only the existence of quantum correlations, but the way they relate to each other, that matters, since the latter can lead to operational interpretations [33]. In fact, figure 22 a) shows that for a time $\tau=10 / \Gamma^{\mathrm{pl}}$, entanglement, as calculated by the EoF, has almost vanished and the quantum discord quickly approximates to the value given by the total correlations. In contrast to this, Ref. [27] uses the concurrence to show that a large amount of entanglement 
is generated for this set of parameters. This can be seen in figure 2/a), where it is evident that the concurrence approximately equals the total correlations, in clear contrast to what is shown by the EoF. Within the formal framework of correlations presented here, the emitters entanglement is well below that reported in Ref. [27]. This is consistent with the approach of entropic measures followed here.

Furthermore, it is straightforward to show that the density matrix computed at $t=\tau$ (figure 2 2a)) corresponds to the mixed state $\rho(\tau)=0.9166|00\rangle\left\langle 00|+0.0834| \Psi^{-}\right\rangle\left\langle\Psi^{-}\right|$, where $\left|\Psi^{-}\right\rangle=(|01\rangle-|10\rangle) / \sqrt{2}$ is the subradiant state. This state has a very small contribution from the entangled state $\left|\Psi^{-}\right\rangle$, and so it provides a small degree of entanglement. However, the mixture of this with the product state $|0\rangle \otimes|0\rangle$, produces a quantum correlated state with a type of correlation - the so-called quantum discord, which is different, in its very essence, to entanglement [15,32].

Although a larger value of $\tilde{\beta}$ can enhance entanglement as well as the other correlations (not shown), we point out that care must be taken when choosing such a parameter; in particular, the value $\tilde{\beta}=0.9$ used in [27] is not realistically attainable according to the plasmon dispersion relation reported there for $d=\lambda_{\mathrm{pl}}$. Indeed, if we include the 'best' achievable $\beta(\simeq 0.94)$ factor, in the expression for $\tilde{\beta} \rightarrow 0.9$, the relation between the plasmonic wavelength and the propagation length should read $\lambda_{\mathrm{pl}} / L \simeq 0.08697$, but from the known dispersion relation [27], no pair $\left\{\lambda_{\mathrm{pl}}, L\right\}$ satisfies this constraint. This simple estimation leads us to conclude that, if $\beta \sim 0.94$, the largest correct realistic value that should be used for the considered emitters separation is $\tilde{\beta} \simeq 0.82$.

The qubit correlation dynamics due to the collective effects arising from the emitters coupling to the plasmon modes, $V^{\mathrm{pl}}$ and $\gamma^{\mathrm{pl}}$, become even more interesting when they are pumped with a continuous laser field of amplitude $\ell_{i}$ and frequency $\omega_{i}$ (targetting the emitter $i$ ). If we consider the laser excitation to be in resonance with the emitters transition frequency $\omega_{i}=\omega_{0}$, stationary correlations can be obtained by making the amplitudes $\ell_{1}=\ell_{2} \equiv \ell$, as shown in figure 2(b) for a distance $\zeta=3 / 4, \gamma^{\mathrm{pl}}=0$. In the same spirit, a higher stationary behaviour of correlations is obtained for $\zeta=1$, by introducing a relative phase between the laser amplitudes: $\ell_{1}=-\ell_{2}=\ell$, as shown in figure 3(a). The correlations are much larger than those obtained in figure 2 (b) because the laser excitation assists the very slow decay of the 'naturally created' antisymmetric state $\left|\Psi^{-}\right\rangle=(|01\rangle-|10\rangle) / \sqrt{2}$. This figure also shows that the concurrence reaches higher values than the total correlations, which might be seen as inappropriate when compared to the use of entropic metrics.

We have shown that rather than entanglement, quantum discord is the most robust correlation during the dissipative dynamics, and, depending on the separation between emitters, the qubit-plasmon modes coupling can enhance the degree of correlations via the collective parameter $\gamma^{\mathrm{pl}}$. For the sake of completeness, in figure 3 (b) we consider, for a fixed laser amplitude, the role of the initial state preparation on the correlations dynamics. Here, we plot the quantum discord as a function of time, for initial states $\sqrt{\alpha}|01\rangle+\sqrt{1-\alpha}|10\rangle$, $\alpha \in \mathbb{R}$. Although it is clear that, at $t=0$, the discord takes the value 1 for $\alpha=1 / 2$, we have plotted it only for a range up to $D=0.3$, in order to appreciate comparatively the correlations behaviour for all $\alpha$. The trend in correlations is clearly influenced by $\alpha$ but, overall, the states converge to a common stationary value. The quantum discord, classical correlations, and EoF are plotted for the particular case $\alpha=1$ in figure $3(\mathrm{c})$, showing how an increment in the laser amplitude may produce a noticeable difference between the quantum discord and entanglement (EoF). Thus, pumping with a continuous laser field can be used as an additional means to dynamically control the relationship between entanglement and the quantum correlations present in the emitters. Such a fact could be used as an important resource in the realisation of non-conventional quantum protocols [17, 18 [3]. 


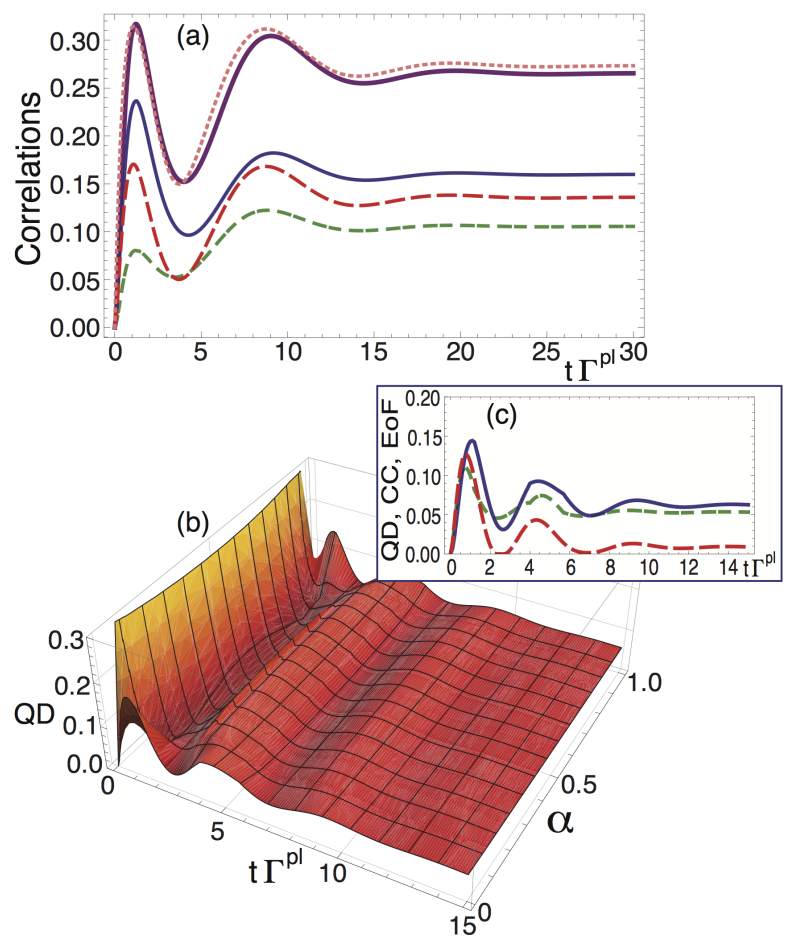

Figure 3. Optical control of correlations dynamics. (a) Total spectrum of correlations for laser amplitude $\ell_{1}=-\ell_{2}=\ell=0.2 \Gamma^{\mathrm{pl}}$, and initial state $|01\rangle$. (b) Quantum discord dynamics for $\ell_{1}=-\ell_{2}=\ell=0.4 \Gamma^{\mathrm{pl}}$, and initial state configurations $\sqrt{\alpha}|01\rangle+\sqrt{1-\alpha}|10\rangle$. (c) Comparison between QD, CC, and EoF for $\alpha=1$. In all graphs, $\zeta=1, V^{\mathrm{pl}}=0, \beta=0.94$, and $L=2 \mu \mathrm{m}$.

Bearing in mind that the quantum correlation, as measured by the discord, gives the amount of information that is not accessible to local measurement, our results show that most of the information stored in the emitters is purely quantum (compare the thin-solid blue with the doubly-dashed green lines in all the figures). Also, we note that, in most cases, this type of information does not arise from entanglement, which is kept to a minimum, as can be seen in Fig.s 1 (b), 2(a), and 3(c). This said, a possible optical control of the amount and class of induced quantum correlations can be carried out with external laser pumping, as depicted in figure 3 .

\section{Discussion}

A comparison of the two physical scenarios considered here - emitters dissipative dynamics in the i) absence and ii) presence of coupling to a plasmonic bus- leads us to the following. In the former case, the correlations exhibit a longer lifetime in figure 1 (b) because of the presence of the incoherent interaction $\gamma$ for the distance $r_{12}=\frac{3}{4} \lambda_{0}$; this means that the lifetime of the symmetric correlated state $\left|\Psi^{+}\right\rangle=(|01\rangle+|10\rangle) / \sqrt{2}$ (an eigenstate of the bare Hamiltonian $\left.H_{S}\right)$ is more robust $(\gamma<0)$. In the latter scenario, $\gamma^{\mathrm{pl}}=0$ and the correlations are only mediated by the coherent interaction $V^{\mathrm{pl}}$. The plasmon-mediated coupled emitters become more interesting because for large distances $\left(d \sim \lambda_{\mathrm{pl}}\right)$, the incoherent decay $\gamma^{\mathrm{pl}}$ can take values close to $\Gamma^{\mathrm{pl}}$, holding the correlations for longer times. A particular case is shown 
in figure 2 (a): $d=\lambda_{\mathrm{pl}}, \gamma^{\mathrm{pl}}=0.82 \Gamma^{\mathrm{pl}}$, hence $V^{\mathrm{pl}}$ is 'switched off'. The correlations are present even for times of the order of $\Gamma^{\mathrm{pl}} t \sim 10$ thanks to the slow decay $\Gamma^{\mathrm{pl}}-\gamma^{\mathrm{pl}}$ of the antisymmetric state $\left|\Phi^{-}\right\rangle$. For this time scale, entanglement (as measured by the EoF) is almost zero, and the correlation that prevails is the quantum discord, which in turn tends to the same value as the total correlations.

Aside from the physics that arises in the qubit-plasmon setup here considered, we highlight the discrepancy between the EoF and the concurrence as quantifiers of the emitters entanglement. This is due to the entropic origin of the EoF compared to that of concurrence (the difference between the two is explicitly stated in equation 2.11). Furthermore, in most of the plots of this work, the concurrence is larger than the mutual information (purple lines) in several different time frames. Since the quantum mutual information captures all the possible correlations (entanglement included), this result seems incorrect. Again, the explanation for this lies in the fact that the concurrence does not have an entropic origin like the other correlation measurements calculated here. Strictly speaking, the concurrence is an entanglement monotone but is not an 'actual' entanglement metric in the sense that it obtains its meaning from its relation to the entanglement of formation and not the opposite [35]. With the emergence of quantum correlations beyond the entanglement, it is crucial to coherently quantify the latter in order to give a consistent physical interpretation of quantum phenomena. The understanding of whether a qubit system is entangled or discord-correlated is thus relevant for deciding the way in which the emitters could be operated as a physical device for performing information processing tasks.

The relation between the correlations and the physical properties of the hybrid system analyzed here is clearly showed in Figs. 113 the lifetime of the correlations is enhanced thanks to the illumination with the coherent laser field; also, a large $\beta$-factor (due to the plasmon channel) increases the degree of correlations, especially that of the quantum discord. It is evident from figure 3 that an adequate manipulation of the light-matter interaction strength allows control of the dynamics followed by the quantum discord and entanglement, and therefore of their metric value. This degree of quantum control is possible due to the interplay between the laser illumination intensity and the coherent emitter-emitter interaction [20, 36].

\section{Concluding remarks}

We have computed entanglement, classical, quantum, and total correlations for a hybrid system composed of largely separated emitters coupled to the plasmonic modes of a onedimensional waveguide, which is externally driven by a laser field. We have illustrated, by direct calculation, that classical correlations are the least assisted by the plasmon bus and that quantum discord is the dominant correlation that prevails throughout the whole dissipative dynamics and that this is enhanced by the presence of the plasmonic collective excitations and by the external laser pumping. This tendency in the correlations has been analytically demonstrated for bare emitters coupled to the vacuum electromagnetic field in Ref. [36].

We have emphasized the entropic origin of the entanglement of formation, the quantum discord, and that of the quantum mutual information as quantifiers of correlations; we also computed the concurrence for direct comparison of our results with those reported in [27, 28]. We found that the latter can reach values well above the EoF and the total correlations. This shows that care must be taken when confronting or using specific quantifiers of entanglement for describing a physical process, especially because the interpretation of the EoF as the cost of creation of an entangled state (with no regularization), leads to an upper bound on the actual degree of entanglement in a quantum system [37]. 
We remark that a crucial feature of the mechanism devised here is the large separation between emitters (of the order of or greater than the plasmons wavelength), which could lead to long-distance quantum control and communication: the correlations are purely generated via the strong plasmon-emitters interaction, which in turn is reflected in the functional dependence of the collective parameters given by equation (2.6). An interesting by-product is the feasibility of tailoring the phase difference that exists between the collective parameters, which might allow the switching on/off of the emitters correlations. Here, a parameter configuration where either $V^{\mathrm{pl}}$ or $\gamma^{\mathrm{pl}}$ goes to zero, an appropriate choice of initial conditions and laser tailoring, enables the enhancement or suppression of the existing correlations. This result is beyond the scope of this work and will be addressed elsewhere [38].

Recent experiments have demonstrated the control of light-matter interaction by means of plasmonic resonators [39] and silver nanowires [40] to enable the enhancement of the Purcell effect in quantum emitters, and the strength of the coupling to the plasmonic modes of such nanostructures. This offers the possibility of testing the results here reported with stateof-the-art technology. Furthermore, another experimental demonstration of communication between two distant single emitters (organic molecules) via single photons has been recently reported [41]. Thus, the setup proposed in this work has also the potential for demonstrating an additional degree of quantum control in which sensitive quantum information encoded in single photons can be transmitted and processed between coupled (but largely separated) single emitters.

\section{Acknowledgments}

C.E.S. is grateful for a Colciencias fellowship. We acknowledge partial financial support from Universidad del Valle (Grant CI 7859), the Spanish DGI (Grant FIS2011-26786), and the UCM-BSCH program (Grant GR-920992).

\section{References}

[1] Horodecki R, Horodecki P, Horodecki M and Horodecki K 2009 Rev. Mod. Phys. 81865

[2] De Martini F, Sciarrino F and Vitelli C 2008 Phys. Rev. Lett. 100253601 Pomarico E, Sanguinetti B, Sekatski P, Zbinden H and Gisin N 2011 New J. Phys. 13063031

[3] Togan E et al. 2010 Nature 466730

[4] Julsgaard B, Koshekin A and Polzik E S 2001 Nature 413400

[5] O’Brien J L, Furusawa A and Vučković J. 2009 Nat. Photonics 3687

[6] Bennett C and DiVincenzo D 2000 Nature 404247

[7] Nielsen M A and Chuang I L Quantum Computation and Quantum Information (Cambridge: Cambridge University Press 2010)

[8] Knill E 2010 Nature 463441

[9] Bennett C H, Brassard G, Crépeau C, Jozsa R, Peres A and Wootters W K 1993 Phys. Rev. Lett. 701895

[10] Gisin N, Ribordy G, Tittel W and Zbinden H 2002 Rev. Mod. Phys 74145

[11] Ladd T D, Jelezko F, Laflamme R, Nakamura Y, Monroe C and O’Brien J L 2010 Nature 46445

[12] Schoelkopf R J and Girvin S 2008 Nature 451664

[13] Benjamin S C, Lovett B W and Reina J H 2004 Phys. Rev. A 70 060305(R)

[14] Makhlin Y, Schön G and Shnirman A 2001 Rev. Mod. Phys. 73357

[15] Ollivier H and Zurek W H 2001 Phys. Rev. Lett. 88017901

[16] Henderson L and Vedral V 2001 J. Phys. A 346899

[17] Knill E and Laflamme R 1998 Phys. Rev. Lett. 815672

[18] Datta A, Shaji A and Caves C M 2008 Phys. Rev. Lett. 100050502

[19] Hettich C, Schmitt C, Zitzmann J, Kühn S, Gerhardt I and Sandoghdar V 2002 Science 298385

[20] Reina J H, Beausoleil R G, Spiller T P and Munro W J 2004 Phys. Rev. Lett. 93 250501; Susa C E and Reina J H 2010 Phys. Rev. A 82042102

[21] Chang D E, Sorensen S, Hemmer P R and Lukin M D 2006 Phys. Rev. Lett 97053002

[22] Reina J H, Quiroga L and Johnson N F 2000 Phys. Rev. B 62 2267(R) 
[23] Reina J H, Quiroga L and Johnson N F 2000 Phys. Rev. A 62, 012305

Lovett B W, Reina J H, Nazir A and Briggs G A D 2003 Phys. Rev. B 68205319

[24] Dzsotjan D, Sorensen S and Fleischhauer M 2010 Phys. Rev. B. 82075427

[25] Dzsotjan D, Kästel J and Fleischhauer M 2011 Phys. Rev. B 84075419

[26] Martín-Cano D, Martín-Moreno L, García-Vidal F J and Moreno E 2010 Nano Lett. 103129

[27] González-Tudela A, Martín-Cano D, Moreno E, Martín-Moreno L, Tejedor C and García-Vidal F J 2011 Phys. Rev. Lett. 106020501

[28] Martín-Cano D, González-Tudela A, Martín-Moreno L, García-Vidal F J, Tejedor C and Moreno E 2011 Phys. Rev. B 84235306

[29] Wootters W K 1998 Phys. Rev. Lett. 802245

[30] Groisman B, Popescu S and Winter A 2005 Phys. Rev. A. 72032317

[31] Hamieh S, Kobes R and Zaraket H 2004 Phys. Rev. A 70, 052325

[32] Modi K, Paterek T, Son W, Vedral V and Williamson M 2010 Phys. Rev. Lett. 104080501

[33] Fanchini F F, Castelano L K, Cornelio M F and de Oliveira M C 2012 New J. Phys. 14, 013027

[34] Zu C et al. 2012 New J. Phys. 14, 033002

[35] Plenio M B and Virmani S 2007 Quant. Inf. Comput. 7, 1

[36] Susa C E and Reina J H 2012 Phys. Rev. A 85022111

[37] Cornelio M F, De Oliveira M C and Fanchini F 2011 Phys. Rev. Lett. 107020502

[38] Susa C E, Reina $\mathrm{J} \mathrm{H}$ and Hildner R, in preparation.

[39] de Leon N P, Shields B J, Yu C L, Englund D E, Akimov A V, Lukin M D and Park H 2012 Phys. Rev. Lett. 108, 226803

[40] Kumar S, Huck A and Andersen U L 2013 Nano Lett. 13, 1221

[41] Rezus Y L A et al 2012 Phys. Rev. Lett. 108, 093601 